\title{
Ophthalmoplegic migraine in a 15-year-old Ethiopian: case report and literature review
}

\author{
Belachew Degefe Arasho
}

Received: 22 July 2008/ Accepted: 2 December 2008/Published online: 8 January 2009

(C) Springer-Verlag 2008

\begin{abstract}
The International Headache Society (IHS) defines ophthalmoplegic migraine $(\mathrm{OM})$ as recurrent attacks of headache with migrainous characteristics, associated with paresis of one or more ocular cranial nerves (commonly the third cranial nerve), and in the absence of any demonstrable intracranial lesion other than MRI changes within the affected nerve. According to the IHS criteria, it is diagnosed when at least two attacks with migraine-like headaches are accompanied with, or followed within 4 days of onset by, paresis of one or more of the third, fourth or sixth cranial nerves. Parasellar, orbital fissure and posterior fossa lesions should be ruled out by appropriate investigations. It is unlikely that $\mathrm{OM}$ is a variant of migraine, since the headache often lasts for a week or more and there is a latent period of up to 4 days from the onset of headache to the onset of ophthalmoplegia. Furthermore, in some cases MRI shows gadolinium uptake in the cisternal part of the affected cranial nerve and this suggests that the condition may be a recurrent demyelinating neuropathy. In general, patients demonstrated a: (1) prolonged time for symptom resolution to occur (median time 3 weeks); (2) tendency for recurrent episodes to have more severe and persistent nerve
\end{abstract}

Electronic supplementary material The online version of this article (doi:10.1007/s10194-008-0089-8) contains supplementary material, which is available to authorized users.

B. D. Arasho $(\bowtie)$

Medical Faculty, Department of Neurology,

Addis Ababa University, Addis Ababa, Ethiopia

e-mail: belachewda@yahoo.com

B. D. Arasho

Headache Medicine, La Sapienza University in Rome,

Rome, Italy involvement; (3) evidence of permanent neurological sequelae with recurrent episodes (30\% of patients); (4) rapid improvement and shortened duration with corticosteroid therapy and; (5) transient, reversible MRI contrast enhancement of the affected cranial nerve $(86 \%$ of patients). Different pathogenetic mechanisms, which include compressive, ischemic and inflammatory, have been suggested for OM. Here, a 15-year-old Ethiopian with recurrent attacks of headache and third nerve palsy is presented. The subsequent discussion focuses on current evidences with regard to the clinical characteristics, possible pathogenetic mechanisms and treatment. Finally, a brief discussion of the situation in Africa will be presented.

Keywords Ophthalmoplegic $\cdot$ Migraine

\section{Presentation of the case}

The patient is a 15 -year-old male who had recurrent attacks of headache for the past 10 years. The pain is left hemicranial, more retro-orbital, throbbing/aching and moderate to severe in intensity. It is associated with photophobia and phonophobia, but not with nausea and vomiting. After the initial attack, he has similar episodes coming every year. Each attack is followed after 2 or 3 days by the drooping of the eyelid on the left side and with difficulty moving the eyes sideways, and diplopia. The weakness lasts for about $2-3$ months and gradually resolves and recovery is complete. He has no associated weakness of the extremities or the face, numbness, abnormal body movements, protrusion of the eye on the affected side or pain on moving the eyes. He has a family history of recurrent headaches: his mother and his paternal grandmother have migraine-like headache, but no abnormality around the eyes. He is the fourth child 
in the family and has five brothers and two sisters, and all are healthy.

When examined during the last attack, four days after the onset of headache, the headache had resolved and the patient was not in any form of pain. His left pupil was $4 \mathrm{~mm}$ and his right $2 \mathrm{~mm}$. His extra-ocular movements were consistent with a complete III nerve palsy with dilated and sluggishly reactive pupil. Fundoscopic examination was unremarkable and there was no exophthalmos. The other cranial nerves including the 4 th and the 6 th were normal. There was no abnormality on motor and sensory examination. Gait and stance were normal. Laboratory evaluation: normal complete blood count and erythrocyte sedimentation rate, the renal and liver function tests were normal, and his blood sugar was normal. Magnetic resonance imaging without contrast administration revealed no mass lesions. Examination 2 months after the onset of the headache showed complete recovery of extra-ocular motor function and there was mild pupillary dilatation.

\section{Discussion}

The international classification of headache disorders classifies ophthalmoplegic migraine (OM) under 'cranial neuralgias and central causes of facial pains' and not under migraine. It does not consider this condition as migraine, but rather as neuralgia [1]. The International Headache Society (IHS) defines OM as recurrent attacks of headache with migrainous characteristics, associated with paresis of one or more ocular cranial nerves (commonly the third cranial nerve) and in the absence of any demonstrable intracranial lesion other than MRI changes within the affected nerve. According to the IHS criteria, it is diagnosed when at least two attacks with migraine-like headaches are accompanied with, or followed within 4 days of onset by, paresis of one or more of the third, fourth or sixth cranial nerves. Parasellar, orbital fissure and posterior fossa lesions should be ruled out by appropriate investigations. Headache is described as migraine like in the ICHD-II, but in most published cases the description is non-existent or poor [2]. But, in the cases of Giraud et al. [2], the description of the pain does not appear different in type, location or intensity from the usual migraine attacks. According to Crevits [3], the type of headache with which occasional episodes of OM are associated varies from case to case and no characteristic headache type has been identified. Accordingly, the above mentioned patient who fulfills all the criteria for the diagnosis of OM (recurrent attacks of migraine like headache, followed in 2-3 days by the third cranial nerve lesion, and absence of other lesions explaining the abnormality on MRI taken without contrast).
Historically, the first case of $\mathrm{OM}$ was attributed to $\mathrm{Gu}$ bler in 1860. The first English language case by Saundby in 1892 described a 19-year-old female with repeated episodes of headache and third nerve palsy. Charcot used the term "migraine ophtalmoplegique" in 1890 and referred to 19 previously published cases [4].

It is a very rare condition. The prevalence is estimated to be 0.7 per million [5-7]. Hansen et al. [5] reviewed the charts of patients admitted from April 1976 to March 1986 to the departments of neurology, neurosurgery, ophthalmology and pediatrics, serving a population of 615,000 inhabitants in Copenhagen County. They then examined patients with coexisting headache and ophthalmoplegia at follow-up. Many diseases may mimic a single attack of OM. They found four cases of OM, i.e., an annual incidence of 0.7 per million inhabitants. In another series of 5,000 cases of migraine headache, only 8 had this uncommon form of headache [8, 9]. In his review on OM, Daroff [4] also noted the rarity of the condition and stated that in the late 1970s he estimated the number of cases with OM to be 1-2 cases per year and he did not see a case in the 14 years before 1994. In a more recent review, Giraud et al. in a retrospective and multicentre database analyzed only nine cases considered as having headaches with oculomotor palsy from among 52,973 cases. According to Carlow [1], over a 7-year period, he examined six children who fulfilled the clinical criteria for ophthalmoplegic migraine. The exception was that all children had abnormal MRI scans at the oculomotor midbrain exit, suggesting a focal pathologic process. In his series, five of six patients were girls. The age of onset varied from 16 months to 9 years, with a mean of 3.7 years. Headache preceded the onset of a third nerve paralysis by up to 11 days. The pupil was dilated in five. Each child had at least 2 and as many as 12 events. Permanent oculomotor paresis or paralysis was present in four of the six children after multiple episodes, and two developed aberrant regeneration. Four of the six children had headaches unassociated with oculomotor paresis [10]. In general, unlike patients with migraine, patients with OM demonstrated: (1) prolonged time for symptom resolution to occur (median time 3 weeks); (2) tendency for recurrent episodes to have more severe and persistent nerve involvement; (3) evidence of permanent neurological sequelae with recurrent episodes (30\% of patients); (4) rapid improvement and shortened duration with corticosteroid therapy and; (5) transient, reversible MRI contrast enhancement of the affected cranial nerve ( $86 \%$ of patients) [11].

It is unlikely that OM is a variant of migraine, since the headache often lasts for a week or more and there is a latent period of up to 4 days from the onset of headache to the onset of ophthalmoplegia [1???]. Furthermore, in some cases MRI shows gadolinium uptake in the cisternal part of the affected cranial nerve and this suggests that the condition may be a recurrent demyelinating neuropathy. Mark et al. [12] 
reported six cases with typical features of $\mathrm{OM}$ in whom enhancement of the cisternal segment of the oculomotor nerve developed during the acute phase followed by resolution of enhancement over several weeks as the symptoms resolved. They concluded that an intrinsic transient abnormality in the cisternal segment of the third nerve had a pathophysiologic mechanism in patients with a typical clinical presentation of ophthalmoplegic migraine. In another study, O'Hara et al. reported on two children with ophthalmoplegic migraine and characteristic magnetic resonance imaging (MRI) findings post enhancement with gadopentetate dimeglumine diethylenetriamine pentaacetic acid (Gd-DTPA). In both cases, enhancement and thickening of the cisternal segment of the oculomotor nerve were present at initial presentation. Ophthalmoplegia resolved spontaneously in one child and after steroid treatment in the other. Both post resolution MRIs demonstrated decrease in enhancement and thickening of the third nerve. They stated that the enhancing, thickened lesion identified on MRI in these patients may represent an inflammatory process similar to Tolosa-Hunt syndrome occurring in the interpeduncular segment of the oculomotor nerve. MRI of the brain post enhancement is a useful adjunct in the diagnosis and treatment of ophthalmoplegic migraine and should be considered as part of a workup of OM in children [13].

Although the oculomotor is the commonly involved nerve in OM, there are case reports for the involvement of the 4th and 6th cranial nerves. Abducens nerve involvement is said to occur in about $10 \%$ of patients with OM [14]. Lee et al. [15] described a patient with $\mathrm{OM}$ and right abducens palsy, who had reversible enhancement of the intraparenchymal part of the nerve with MRI during both the headache and headache-free periods. They also showed with thin MRI sections that gadolinium enhancement of the posterior brainstem area corresponds to the intraparenchymal pathway of the abducens nerve. This area is also the location of the raphe nuclei, which regulate serotonin production, a crucial neurotransmitter in migraine. Verhagen et al. [16] described a patient from The Netherlands, who had onset in middle age of features of $\mathrm{OM}$ with abducens palsy. Vasconcelos [17] also reported on a 40year-old female patient from Brazil with OM and recurrent abducens palsy. Van der Dussen et al. [18] reported a case of recurrent ophthalmoplegic migraine with oculomotor and trochlear palsies and reversible MRI enhancement and thickening of the trochlear nerve.

\section{Possible pathogenesis of ophthalmoplegic migraine}

A number of theories have been suggested for the mechanism of ophthalmoplegic migraine [19]. Stommel et al. [19] suggested an ischemic mechanism of ophthalmoplegic migraine, given the MRI findings of enhancement of the third cranial nerve at the point of exit from the brainstem. However, the third cranial nerve did not enhance on MRI imaging in patients with diabetic oculomotor palsy, even though this condition is thought to be due to ischemia [20]. Shin et al. also suggested a possible ischemic mechanism for OM. They evaluated two patients with OM with electroencephalography, brain MRI, angiography and SPECT during an attack. Follow-up SPECT was also performed after the neurologic symptoms resolved. In both patients, SPECT during an attack of ophthalmoplegia and headache demonstrated significantly decreased regional cerebral blood flow in the thalamus on the side of the ophthalmoplegia, which reverted to normal on the follow-up examination during symptomfree periods. They concluded that reversible ischemia in the territories of the perforating branches of the posterior cerebral artery may accompany OM and possibly bear some relationship to the clinical features [21]. An ischemic mechanism has also been proposed secondary to reduced blood flow in the appropriate circulation to ocular motor nerves. In fact, about two-thirds of patients with ophthalmoplegic migraine involving the oculomotor nerve have at least partial pupillary sparing [9, 22]. Vijayan [22] reported on a patient with $\mathrm{OM}$ and oculomotor palsy with pupillary sparing and suggested a possible ischemic mechanism. They stated that most of the clinical evidence suggests that the ophthalmoplegia of OM is due to a delayed ischemic neuropathy. This results from swelling of the walls of the carotid and/or basilar arteries leading to occlusion of the ostia of the smaller vessels, which supply the involved cranial nerves. The course and evolution of ophthalmoplegia suggest that this ischemia leads to a border zone infarction of the nerves. This is very similar to the ischemic neuropathy associated with diabetes. Nevertheless, pupillary sparing does sometimes occur with compressive lesions of the oculomotor nerve such as aneurysms in the cavernous sinus. Penetration of the oculomotor nerve by the circumflex mesencephalic artery or by a branch of the perforating vessels of the posterior cerebral artery occurs frequently [23]. Vasospasm or dilatation of these vessels during migraine attacks could infarct the perimesencephalic portion of the oculomotor nerve. The long circumflex mesencephalic artery supplies the dorsolateral midbrain, crus cerebri, substantia nigra, red nucleus, medial lemniscus, spinothalamic tract, reticular formation, superior colliculi and portions of the pretectal and diencephalic regions; thus ischemia in the distribution of such a vessel could result in hemiparesis, supranuclear facial and genioglossus paresis, extrapyramidal disturbances and ataxia in addition to ophthalmoplegia[19].

The compressive theory for ophthalmoplegic migraine suggests that one or more of the ocular motor nerves is compressed by an edematous or dilated intracavernous portion of the carotid artery. Walsh and O'Doherty [24] 
suggested that cavernous carotid artery enlargement occurred with the compression of the intracavernous cranial nerves. Vieira et al. [25] reported on a 7-year-old boy with typical symptoms of OM, whose angiography (both magnetic resonance and computerized tomography angiography) revealed the presence of an infundibular dilatation of a perforating branch of the posterior cerebral artery adjacent to the symptomatic third cranial nerve. They speculated that the contact between the third nerve and the dilated intracranial artery could episodically produce a migrainous syndrome sustained by activation of the trigeminovascular system as well as compression and paralysis of the third nerve. Others proposed an infectious or inflammatory pathogenesis for ophthalmoplegic migraine [11, 26, 27]. Lance and Zagami [28] consider the possibility that ophthalmoplegic migraine is a recurrent neuritis or demyelinating neuropathy. An inflammatory process could trigger migraine headaches in people who are already migraineurs. Discussing the findings of four cases with OM, Lance and Zagami noted that the MRI appearances of the enhancement of the oculomotor nerve, as it emerges from the midbrain, favors demyelination or inflammation of the oculomotor nerve in cases of "ophthalmoplegic migraine", rather than an ischemic lesion. The most striking feature of the usual MRI changes is the globoid appearance of the first part of the oculomotor nerve on emerging from the midbrain, indicating a gross localized enlargement of the nerve, consistent with intraneural edema as reported in some experimental demyelinating neuropathy and in chronic inflammatory demyelinating polyneuropathy (CIDP) [28].

\section{Differential diagnosis of ophthalmoplegic migraine}

A number of other conditions that are similar to ophthalmoplegic migraine can present; hence, exclusion of vascular, neoplastic, infectious and inflammatory causes must be the first step in the workup of these patients [14]. These include aneurysm (generally of the posterior communicating artery), tumor or abscess in the region of the cavernous sinus or superior orbital fissure, Tolosa-Hunt syndrome, sphenoid sinus pathology, diabetic cranial nerve 3,6 or 4 dysfunction, nerve sheath tumor of cranial nerves 3 , 6 or 4, and meningitis or other inflammatory processes such as Lyme disease, sarcoidosis, fungal infection and tuberculosis. Cavernous sinus syndrome is suggested by upper facial (and corneal) hypesthesia and Horner's syndrome [14].

\section{Treatment}

The rarity of OM has made a comprehensive study of treatment difficult. Prophylactic treatment seems warranted to prevent the development of permanent ocular palsies seen in OD. Calcium channel blocker or $\beta$-blocker medication is appropriate if the pathophysiology is akin to migraine. Some authors have recommended standard migraine prophylactic therapy with $\beta$-blocker or calcium channel blocker medication for patients with recurrent attacks [14]. Although there are no controlled studies on the effect of steroids, some authors conclude that prompt administration of steroids at the time of attack might minimize permanent sequelae of $\mathrm{OM}$, including residual weakness of the third cranial nerve and pupillary dysfunction [29].

\section{Ophthalmoplegic migraine from African studies}

Like studies done in other parts of the world, African studies have shown a very small incidence of OM. Okogbo [30] evaluated 51 patients to determine the profile of migraine in Nigerian children and commented that complicated migraine phenomenon such as hemiplegia or ophthalmoplegia were not observed, although migraine complicated by a hemiplegic or hemisensory aura is thought to be commoner in children than in adults. It is possible that some of the cases of unexplained hemiplegia in their clinic may be of migrainous origin. A long-term follow-up may help resolve this.

In a study to determine the causes of severe headache in Sierra-Leone, Lisk [31] evaluated 250 indigenous Sierra-Leoneans with different forms of headache and found out that migraine was the most common cause of severe headache and accounted for $40 \%$ of all cases. There was only one case, which could either be ophthalmoplegic or hemiplegic (according to the 1962 ad hoc committee classification of headache disorders, which lumped hemiplegic and ophthalmoplegic migraine together). This study also shows the rarity of $\mathrm{OM}$ in African populations.

In Ethiopia, there are no case reports or other studies on $\mathrm{OM}$, and the prevalence or incidence of the condition is not known. In his 1995 extensive study on the chronic forms of headache in the rural community of Ethiopia, Tekle-Haimanot [32] did not mention anything about the occurrence of OM. It may be because those patients with presumed $\mathrm{OM}$ were lumped under migraine with aura category because $\mathrm{OM}$ was classified under migraine in the first international classification of headache disorders [33].

So, I believe that this case report would add to the understanding of $\mathrm{OM}$ in our setup and open the door for further community-based studies to look into the situation in Africa and if there are any differences from those of other countries. 
Acknowledgments This work represents the final dissertation held at the Master in Headache Medicine by Dr. Belachew Degefe Arasho, during the Academic Year 2007-2008 at the 2nd School of Medicine, Sapienza University of Rome. Dr. Arasho's scholarship was granted by Sapienza University (0010959-3.1.6.1.1.10 to Paolo Martelletti) in favor of the Working Group of Specialist Education, WHO Global Campaign Lifting the Burden.

Conflict of interest None.

\section{References}

1. Classification Committee of the International Headache Society (2004) International classification of headache disorders II. Cephalalgia 24(suppl 1):1-160

2. Giraud P, Valade D, Lanteri-Minet M, Donnet A, Geraud G (2007) Is migraine with cranial nerve palsy and ophthalmoplegic migraine. J Headache Pain 8:119-122

3. Crevits L, Verschelde H, Casselman J (2006) Ophthalmoplegic migraine: an unsolved problem. Cephalalgia 26:1255-1259

4. Daroff RB (2000) Random comments, neurologists and neuroophthalmology: the ocular motor system; update on ophthalmoplegic migraine. Sem Neurol 20:145-149

5. Hansen SL, Borelli-Moller L, Strange P et al (1990) Ophthalmoplegic migraine: diagnostic criteria, incidence of hospitalization, and possible etiology. Acta Neurol Scand 81:54-60

6. O'Halloran HS, Lee WB, Baker RS, Pearson PA (1999) Ophthalmoplegic migraine with unusual features. Headache 39:670673

7. Quisling S, Kardon RH (2006) Ophthalmoplegic migraine: 30year-year old male with migraine headaches and occasional diplopia. Eyerounds Org

8. Arunagiri G, Santhi S (2003) Migraine: an ophthalmologist's perspective. Curr Opin Ophthalmol 14:344-352

9. Friedman A, Harter DH, Merrtitt HH (1962) Ophthalmoplegic migraine. Arch Neurol 7:320-327

10. Carlow TJ (2002) Oculomotor ophthalmoplegic migraine: is it really migraine. J Neuro-Ophthalmol 22:215-221

11. McMillan HJ, Keene DL, Jacob P, Humphreys P (2007) Ophthalmoplegic migraine: inflammatory neuropathy with secondary migraine? Can J Neurol Sci 34(3):349-355

12. Mark AS, Casselman J, Brown D et al (1998) Ophthalmoplegic migraine: reversible enhancement and thickening of the cisternal segment of the oculomotor nerve on contrast-enhanced MR images. AJNR Am J Neuroradiol 19:1887-1891

13. O'Hara MA, Anderson RT, Brown D (2001) Magnetic resonance imaging in ophthalmoplegic migraine of children. J AAPOS 5(5):307-310

14. Levin M, Ward TN (2004) Ophthalmoplegic migraine. Curr Pain Headache Rep 8:306-309
15. Lee TG, Choi WS, Chung KC (2002) Ophthalmoplegic migraine with reversible enhancement of intraparenchymal abducens nerve on MRI. Headache 42:140-141

16. Verhagen WI, Prick MJ, Azn RD (2003) Onset of ophthalmoplegic migrainewith abducens palsy at middle age? Headache 43:798-800

17. Vasconcelos LP, Stancioli FG, Leal JC, da Silva A, Gómez RS, Teixeira A (2008) Ophthalmoplegic migraine: a case with recurrent palsy of the abducens nerve. Headache 48(6):961-964

18. van der Dussen D, Bloem BR, Liauw L, Ferrari MD (2004) Ophthalmoplegic migraine: migrainous or inflammatory? Cephalalgia 24:312-315

19. Stommel EW, Ward TN, Harris RD (1993) MRI findings in a case of ophthalmoplegic migraine. Headache 33:234-237

20. Blake PY, Mark AS, Kattah J et al (1995) MR of oculomotor nerve palsy. AJNR Am J Neuroradiol 16:1665-1672

21. Shin DJ, Kim JH, Kang SS (2002) Ophthalmoplegic migraine with reversible thalamic ischemia shown by brain SPECT. Headache 42:132-135

22. Vijayan N (1980) Ophthalmoplegic migraine: ischemic or compressive neuropathy? Headache 20:300-304

23. Milisavljevic M, Marinkovic S, Lolic-Draganic V, Kovacevic M (1986) Oculomotor, trochlear, and abducens nerves penetrated by cerebral vessels. Arch Neurol 43:58-61

24. Walsh JP, O'Doherty DS (1960) A possible explanation of the mechanism of ophthalmoplegic migraine. Neurology 10:10791084

25. Vieira JP, Castro J, Gomes LB, Jacinto S, Dias A (2008) Ophthalmoplegic migraine and infundibular dilatation of a cerebral artery. Headache 48:1372-1374

26. Wong V, Wong WC (1997) Enhancement of oculomotor nerve: a diagnostic criterion for ophthalmoplegic migraine? Pediatr Neurol 17:70-73

27. Prats JM, Mateos B, Garaizar C (1999) Resolution of MRI abnormalities of the oculomotor nerve in childhood ophthalmoplegic migraine. Cephalalgia 19:655-659

28. Lance JW, Zagami AS (2001) Ophthalmoplegic migraine: a recurrent demyelinating neuropathy? Cephalalgia 21:84-89

29. Bharucha DX, Campbell TB, Valencia I et al (2007) MRI fi ndings in pediatric ophthalmoplegic migraine: a case report and literature review. Pediatr Neurol 37:59-63

30. Okogbo ME (2005) Migraine in Nigerian children: a study of 51 patients. Headache 31:673-676

31. Lisk DR (2005) Severe headaches in the African: Report on 250 cases from Sierra-Leone, West Africa. Headache 27:477-483

32. Tekle Haimanot R, Seraw B, Forsgren L, Ekbom K, Ekstedt J (1995) Migraine, chronic tension type headache, and cluster headache in an Ethiopian rural community. Cephalalgia 15:482488

33. Headache Classification Subcommittee of the International Headache Society (1988) Classification and diagnostic criteria for headache disorders, cranial neuralgias and facial pain. Cephalalgia 8(Suppl. 7):1-96 